\title{
1 Genomic insights into evolutionary journey of the porcine
}

\section{2 endogenous retroviruses}

3

4 Yicong Chen ${ }^{1,2}$ ฯ, Mingyue Chen ${ }^{1 \uparrow}$, Xiaoyan Duan ${ }^{1,2}$, Jie Cui $^{1}$ *

5

$6 \quad{ }^{1}$ CAS Key Laboratory of Special Pathogens and Biosafety, Center for Emerging

7 Infectious Diseases, Wuhan Institute of Virology, Chinese Academy of Sciences,

8 Wuhan 430071, China.

$9 \quad{ }^{2}$ University of Chinese Academy of Sciences, Beijing 100049, China.

10

11 *Corresponding author

12 E-mail: jiecui@wh.iov.cn

13

14 ๆ Y.C. and M.C. contributed equally to this work 


\section{Abstract}

16 Background: Xenotransplantation may overcome significant shortage of human

17 allotransplant. Porcine organs are considered favorable for xenotransplantation duo to

18 similar size and function to human organ. However, porcine endogenous retroviruses

19 (PERVs) are potential infectious agents during xenotransplantation as they are able to

20 infect and horizontally transfer among human cells. Furthermore, PERVs can be

21 endogenized in pig genomes and are transmitted genetically in a Mendelian fashion. Here,

22 we depict a complex evolutionary history of modern PERVs.

23 Results: We in silico mined 142 mammalian genomes and 14 pig genomes. This led to the

24 documentation of 185 PERVs and a new viral cluster. Large-scale genomic alterations

25 were found in most PERVs including many insertion-deletion events and which are

26 suggestive of ancient origins, and pig genomes have been shaped by PERV-mediated

27 genomic rearrangement during evolution. Notably, we found that lesser Egyptian jerboa

28 and rock hyrax harbor ancestral PERV-related elements indicative of ancient cross-species

29 transmission events from none-porcine species to pigs. A comprehensive analysis of these

30 viral "fossils" suggested that recombination among none-porcine endogenous retroviruses

31 led to the origination of PERVs.

32 Conclusion: For the first time, using large scale genomic mining, we decipher a complex

33 evolutionary history for the PERVs. These new findings help us to understand the past of

34 PERVs which pose the potential risk in clinical trials of xenotransplantation and provide

35 novel insights into the origin and evolution of a human-infecting pathogen. 
37 Keywords: porcine endogenous retrovirus, genomic rearrangement, cross-species

38 transmission, evolution, origin, recombination

\section{Background}

41 Xenotransplantation, the transplantation of tissues and organs from one species to another,

42 may alleviate shortages of human donor $\operatorname{organs}(1,2)$. Porcine organs are suitable for

43 xenotransplantation due to their similar size and function to human organs, and the fact

44 that pigs can be bred in large numbers(3). However, porcine endogenous retroviruses

45 (PERVs) are able to infect and horizontally transfer among human cells, raising major

46 concerns about the safety of xenotransplantation(3,4), particularly given that retroviruses

47 are often associated with severe infectious disease or oncogenic disease(3, 5-7).

49 PERVs are endogenous gammaretroviruses, and exist in the genomes of all pig strains(3,

50 8). The envelope (env) genes of three PERV classes (PERV-A, -B and -C) differ with

51 respect to the receptor-binding domain (RBD)(9). Although there is no evidence of PERV

52 transmission in patients receiving encapsulated pig islets(10-12), PERV-A and -B have

53 been observed to infect both human cells and pig cells while PERV-C infects only pig

54 cells(13). PERVs may also integrate into the human genome in vitro(14, 15). In pig cells,

55 PERV-C can recombine with the $e n v$ of PERV-A to produce A/C recombinants, which can

56 infect human cells more efficiently(13). This increases the inherent risk in

57 xenotransplantation and xenogeneic cell therapies. 
59 While several studies have examined the evolutionary relationships between PERVs and

60 other viruses, the origin and evolution of PERVs remains uncertain, particularly why they

61 are unable to infect humans $(16,17)$. At least two species that belong to the same order as

62 pigs, Tayassu pecari (of Eocene origin) and Babyrousa babyrussa (of Miocene origin)

63 lack PERVs(18). However, the common warthog (Phacochoerus africanus) carries

64 PERVs, suggesting that an ancestral porcine species carried PERVs(18). PERVs have two

65 different types of long terminal repeat (LTRs), one with a 39-bp repeat structure in the U3

66 region, and the other without this repeat structure $(19,20)$. The 39-bp repeats carried by

67 PERV-A and -B confer strong promoter activity and thus increase transcription(19, 20).

68 However, the 39-bp repeat structure is absent in some PERV-A and all PERV-C. These

69 PERVs thus have low transcriptional activity $(19,20)$. BLAST search analysis confirmed

70 that the R and U5 regions of the PERV LTRs are highly conserved in the pig and mouse

71 genomes (74-87\% identity)(21). Indeed, the LTRs of PERV-A, -B and LTR-IS (a LTR

72 family found solely in the mouse genome) have similar structures(21). The conserved LTR

73 sequences found in pigs and mice might have originated from a common exogenous viral

74 element, but evolved independently(21). Using genome mining with all available

75 mammalian genomes, we clearly illustrate the evolutionary journey of the modern PERVs

76 and reveal that the genesis of PERVs is much more complex than previously thought.

\section{$78 \quad$ Results}

79 In silico characterization of putative PERVs.

80 Using previously reported PERV sequences as queries, we mined 14 pig genomes 
81 (Additional files 2: Table S1) available in GenBank, and showed a detailed genome-wide

82 distribution of full-length PERVs (i.e. containing two LTRs). We initially compiled a

83 PERV data set that included 185 putative PERVs (containing at least one LTR) (Additional

84 files 2: Table S2). A total of 84 classified (30 PERV-A, 39 PERV-B and 15 PERV-C), 18

85 unclassified full-length PERVs (i.e., lacking of env gene) were retrieved from pig

86 genomes. We identified 2-10 full-length PERVs in each of 12 other pig breeds, including

87 Meishan, Goettingen, and Large White. We removed 19 previously classified PERV

88 sequences that were low quality fragments (> 200 "N" bases). Hence, the final data set

89 comprised 65 high quality classified PERVs (27 PERV-A, 29 PERV-B, and 9 PERV-C).

90 Their viral genomic structures are summarized in Figure 1.

92 Most of the PERVs exhibited large-scale genetic alterations induced by indels and stop

93 codons (Fig. 1), suggestive of a relatively long evolutionary history. PERV LTRs were

94 classified by the presence (LTR B) or absence (LTR A) of the 18 bp and 21 bp repeat

95 structure reported previously $(9,19,22)$. Three different type B LTRs in the PERV were

96 identified, distinguished by the number of $18 \mathrm{bp}$ and $21 \mathrm{bp}$ repeat sequences: LTR B1 (two

97 18-bp and one 21-bp repeats), LTR B2 (three 18-bp and two 21-bp repeats), and LTR B3

98 (four $18 \mathrm{bp}$ and three $21 \mathrm{bp}$ repeats). Of the 65 high-quality PERVs we analyzed, we

99 assigned 57, of which 32 (>55\%) carried LTR A, 10 carried LTR B1, 13 carried LTR B2,

100 and 2 carried LTR B3. LTR A was identified in PERV-A and -C, and LTR B1 was

101 identified in PERV-A and -B. LTR B2 and LTR B3 were only identified in PERV-B. The

102 remaining eight PERVs contained different types of 5'- and 3'- LTR which may reflect 
103 recombination between PERVs with different LTR types (Fig. 1). For example, we

104 discovered one PERV (AEMK02000536.1) with LTR B2 at the 5' end and LTR A at the 3'

105 end (Fig. 1).

\section{Potential genomic rearrangement via PERVs.}

108 Retrovirus integration creates a short duplication called target site duplication (TSD)

109 flanking the $\operatorname{LTR}(23,24)$. If chromosomal rearrangement through homologous

110 recombination between distant proviruses occurred, the flanked TSDs should be different,

111 as mentioned in a previous study of primate genomic rearrangement via ERVs (25). To

112 identify pig genomic rearrangement via PERVs, we firstly constructed a maximum

113 likelihood (ML) tree representing the 5'- and 3'- LTR sequences of full-length PERVs

114 (Additional files 1: Fig. S1). The phylogenetic tree was divided into three large clusters

115 (Additional files 1: Fig. S1), suggesting that three major integration events had occurred.

116 We collected PERVs with 5'- and 3'- LTR sequences did not clustered together in the

117 phylogenetic. Remarkably, 11 PERVs did not share the same TSD (4bp in length) (Table 1,

118 Additional files 2: Table S3), thus these PERVs could reflect porcine genomic 119 rearrangement during evolution.

121 Table 1. PERVs with different target site duplications (TSDs).

\begin{tabular}{lcccccc}
\hline Name & Accession number & $\begin{array}{c}\text { Divergent } \\
\text { LTRs based } \\
\text { on structure }\end{array}$ & $\begin{array}{c}\text { Divergent } \\
\text { LTR based } \\
\text { on tree }\end{array}$ & & Flanking TSD \\
\cline { 5 - 7 } & & AEMK02000536.1 & yes & yes & AGCC & CTTT \\
\hline AEMK02000536.1 & AET & & & $3^{\prime}$ \\
CM000818.5_a & CM000818.5 & no & yes & GTTC & CTTC
\end{tabular}




\begin{tabular}{llllll} 
CM000826.5_c & CM000826.5 & no & no & ACCA & AATC \\
CM000828.5_d & CM000828.5 & no & yes & CCAC & CACC \\
KQ001967.1 & KQ001967.1 & no & yes & CCAC & CACC \\
LIDP01000017.1 & LIDP01000017.1 & no & yes & CCAC & CACC \\
LUXR01004647.1 & LUXR01004647.1 & yes & yes & GTTC & CTTC \\
LUXR01022139.1 & LUXR01022139.1 & yes & yes & CCAC CACC \\
LUXX01045907.1 & LUXX01045907.1 & no & yes & CCAC CACC \\
LUXX01080744.1 & LUXX01080744.1 & no & yes & GTTC & CTTC \\
LUXY01101100.1 & LUXY01101100.1 & no & yes & CCAC & CACC \\
\hline
\end{tabular}

122 *LTRs of PERVs comprise four types (LTR A, B1, B2, and B3). If two different types of

123 LTRs are flanking the PERV, the LTRs are considered "divergent".

$124{ }^{* *}$ Only TSDs flanking the intact 5' and 3' LTRs sequences were analyzed

126 Detection of PERV-related sequences in mammalian genomes.

127 After screening 142 mammalian genomes (Additional files 2: Table S4) available on

128 GenBank using tBLASTn and choosing three major proteins (Gag, Pol and Env) of

129 PERVs as queries, a significant sequence (accession number: NW_004504334.1) in the

130 genome of lesser Egyptian jerboa (Jaculus jaculus) that exhibited strong sequence

131 similarity (for gag and pol: $>75 \%$ nucleotide identity over 95\% region; for $e n v:>75 \%$

132 nucleotide identity over 55\% region) to PERVs. Using this PERV-related sequence as

133 query, three other possible PERV related sequences were identified in J. jaculus (accession

134 number: NW_004504375.1, NW_004504378.1, and NW_004504445.1) with >85\%

135 nucleotide identity over $80 \%$ of the query sequence. The four PERV-related sequences

136 identified in J. jaculus were designated as eJJRVs. These four significant hits located in

137 large scaffolds $>5 \mathrm{Mb}$ in length (Additional files 2: Table S5), with several host genes 
identified, indicated that the eJJRV sequences were relatively reliable.

We were only able to identify one pair of eJJRV LTRs. This full-length eJJRV (containing 2 LTRs) (accession number: NW_004504334.1) is annotated in Additional files 1: Fig. S2. The length of 3'- LTR of this eJJRV is 674 bp while 5'- LTR is 932 bp with a 258 bp insertion. We aligned eJJRV LTRs with PERV LTRs. The start of the U3 region and the end of the U5 region were distinct and not included in the alignment (Additional files 1: Fig. S3). The eJJRV LTRs included a repeat structure (three 18 bp and two 21 bp repeat sequences) in the U3 region, identical to that of the PERV LTR B2. Furthermore, 3' LTR of eJJRV had high identity with LTR B2 of PERV ( 73\%). Alignment analysis revealed a closer relationship between LTRs of the eJJRV and LTR B2 of PERVs (Additional files 1: Fig. S3). Notably, the alignment of the conserved R region supported a close evolutionary relationship between the eJJRV and PERVs (Fig. 2a). To highlight the similarity between PERVs and eJJRVs we generated pairwise alignments of eJJRV and PERV nucleotides using the full-length ERVs, and performed a sliding window analysis of these pairwise alignments (Fig. 2b)(26, 27). For comparison, we determined the similarity of HIV-1 provirus sequence to that of its closest relative (chimpanzee SIVcpz) (28, 29). Interestingly, gag and pol were more similar between eJJRV and PERV-A, -B and -C than HIV-1 and SIVcpz (Fig. 2b). Therefore, our results indicate that eJJRVs and PERVs are homologous. However, the RBD and the proline rich-region (PRR) of the surface subunit (SU) of env were dissimilar between eJJRV and PERV-A, -B and -C, as also seen in HIV and SIVcpz. As RBD determined host range(30-33), this observation suggests that eJJRVs and PERVs have distinct host ranges. 
161 We used the RBD amino acid sequences from PERV-A, -B and -C as queries to screen for

162 homologous viral elements. The eight significant hits (>60\% amino acid identity over $80 \%$

163 region) were obtained in rock hyrax (Procavia canpensis) of Procaviidae, and all eight

164 hits flanking with genes located in large scaffolds $>0.3 \mathrm{Mb}$ in length (accession number:

165 KN678690.1, KN676491.1, KN678005.1, KN677924.1, KN676905.1, KN676182.1,

166 KN680906.1, and KN676638.1) (Additional files 2: Table S5). We examined the

167 sequences flanking the eight hits (especially pol), and found that ERVs including these hits

168 were endogenous gamma-retroviruses. These hits were therefore designated ePCRVs. We

169 aligned the RBDs of PERVs and ePCRVs, and found that ePCRVs were highly similar to

170 PERVs (Fig. 3). Pairwise comparisons revealed that ePCRV_1 and ePCRV_2 had a high

171 identity to PERV-B (63\%) but a low identity with PERV-A, -C and PERV-IM (40-43\%).

172 Therefore, the RBD of ePCRVs and PERV-B are homologous.

174 To characterize the relationship within eJJRVs, ePCRVs and PERVs, we inferred

175 phylogenetic trees of Gag, Pol and Env, first removing the variable RBD (Fig. 2c-e). Our

176 maximum likelihood (ML) phylogenetic tree revealed that eJJRVs and PERVs clustered

177 together with high bootstrap supports in three phylogenies (Fig. 2c-e), suggesting that they

178 shared the most recent common ancestry. However, the Gag of ePCRVs clustered with

179 eJJRVs and PERVs while Pol and Env (without RBD) of ePCRVs were distant related to

180 eJJRVs and PERVs which might indicate recombinant events among ancient retroviruses

181 (Fig. 2c-e). 
183 Remarkably, a new lineage close to PERV-A and PERV-C was observed, named PERV-IM

184 (as designation of PERV-intermediate type), and presented in all 14 pig genomes (Fig. 2e,

185 Additional files 2: Table S2). The Env proteins of PERV-IMs showed relatively low

186 similarity to PERV-A, -B, and -C, and they were clearly distinct in the RBD region (Fig. $1873)$.

189 Discussion

190 Using systematic large-scale genome mining, we analyzed the origin and evolution of the

191 modern PERVs. We found homologous LTRs of PERVs ( 73\% identity) in eight

192 Muroidea species (Mus caroli, M. pahari, M. musculus, M. spretus, Apodemus speciosus,

193 A. sylvaticus, Rattus norvegicus, and Phodopus sungorus). The coding genes (gag, pol,

194 and $e n v$ ) near these homologous LTRs were identified (Additional files 2: Table S6). Also,

195 ERVs in two Muroidea species (M. musculus and R. norvegicus) found in previous study

196 were also presented(34). However, phylogenetic analysis of the Gag (>220 amino acids),

197 Pol (>560 amino acids) and Env (>330 amino acids) sequences of these ERVs suggested

198 that they were distantly related to the modern PERVs (Additional files 1: Fig. S7). We

199 provided robust phylogenetic results that eJJRV shared the most recent ancestry with the

200 PERVs (Fig. 2), and ePCRV also contributed the origin of Gag (Additional files 1: Fig.

201 S7). Failure to detect any other eJJRV and PERV-related elements in the remaining rodent

202 genome screening suggests that the virus was not vertically transmitted, suggesting

203 ancient horizontal transmission occurred during evolution. 
205 PERVs, eJJRVs, and ePCRVs integrated into Suidae, Dipodidae, and Procaviidae,

206 respectively. Miocene (23 - 5.33 MY) Suidae fossils have been found in East Africa,

207 Europe and Asia (http://fossilworks.org/?a=taxonInfo\&taxon_no=42381). Miocene

208 Dipodidae fossils have been found in North Africa, Europe and Asia

209 (http://fossilworks.org/?a=taxonInfo\&taxon_no=41695); Pliocene $(5.3-2.59$ MY)

210 Dipodidae fossils have been identified in East Africa, thus suggesting that the Dipodidae

211 may have spread to East Africa during the Miocene. Miocene Procaviidae fossils have

212 been found in South of Africa and East Africa

213 (http://fossilworks.org/?a=taxonInfo\&taxon_no=43293). According to the current fossil

214 records, the only shared region for Miocene Dipodidae, Procaviidae and Suidae fossils is

215 East Africa. It is likely that ancestral PERV might be derived from two ancient retroviruses

216 carried by Dipodidae and Procaviidae, denoted as JJRV and PCRV in the illustration (Fig.

217 4), in East Africa during the Miocene time. The ancestral PERV then split into different

218 classes (PERV-A, -B, -IM and PERV-C), in which the PERV-B diverged earlier than other

219 classes of PERVs (Fig. 4).

221 We also discovered a new class of PERV named PERV-IM and they are presented in all

222 pig genomes we scanned (Additional files 2: Table S1). It remains unknown whether

223 PERV-IMs are able to infect human cells. However, it is possible that non-human trophic

224 PERV was able to participate in recombination, leading to human trophic retroviruses(35,

225 36). The identification of the new PERVs is crucial for future assessment of pathogen 
226 transmission during xenotransplantation.

228 Conclusions

229 For the first time, we decipher a complex evolutionary history for the PERVs. The 230 ancestral PERV might be derived from two ancient retroviruses carried by non-porcine

231 species. We also suggest that pig genomes have been shaped by PERVs, as specifically

232 reflected by PERV-associated genomic rearrangement that have occurred during porcine

233 evolution. In a word, modern PERVs have a complex evolutionary history than previously

234 thought prior to their appearance in pigs.

\section{Materials and methods}

237 In silico identification of PERV and PERV-related proviruses.

238 To identify PERV-related elements in Sus scrofa, tBLASTn (37) was used and the amino

239 acid sequences of Gag, Pol and Env of 20 representative PERV proviruses (accession

240 numbers: HQ536016.1, HQ536015.1, HQ536013.1, KC116220.1, AY570980.1,

241 HQ540592.1, HQ536007.1, AX546209.1, AF435967.1, AY953542.1, HQ540591.1,

242 AY099323.1, AJ133817.1, EU523109.1, EF133960.1, AY056035.1, AY099324.1,

243 A66553.1, HQ536011.1, and HQ536009.1) were chosen as queries to screen the 14 pig

244 genomes available in GenBank that were released before November 2017. A 50\% identity

245 over $50 \%$ of the match region was used to filter significant hits. It has been shown that

246 PERVs harbor two LTR structures, one with and one without a repeat structure in the U3

247 region $(9,19)$. Using two typical LTRs as queries we extended flanking sequences of 
248 coding domains of PERVs to identify LTRs with BLASTn, and TSDs were used to define

249 PERV boundaries. LTR lengths were defined as 100-1,000 bp. PERVs with at least one

250 LTR and one coding gene were used in the evolutionary analysis.

252 To identify PERV-related proviruses in mammals, tBLASTn was used with the queries

253 described above in 20 representative PERV proviruses to search the 142 mammal genomes

254 available in GenBank as of November 2017. A $50 \%$ identity over $80 \%$ region was used to

255 filter significant hits. LTRs were identified using LTR finder(38), LTRharvest (39) and

256 BLASTn. LTR lengths were also defined as 100-1,000 bp.

\section{Detection of potential genomic rearrangement via PERVs.}

259 To search for proviruses involved in recombination and genomic rearrangement, we

260 constructed a maximum likelihood (ML) tree of the 5'- and 3'- LTRs of full-length PERVs

261 using PhyML 3.1(40) with GTR $+\mathrm{I}+\Gamma$ nucleotide substitution model. LTRs less than 250

262 bp were not considered. Sequence alignment was performed with MAFFT 7.222(41).

\section{Phylogenetic analyses.}

265 To determine the evolutionary relationship among PERVs, eJJRVs, ePCRVs and

266 representative gammaretroviruses (Additional files 2: Table S7), phylogenetic trees were

267 inferred using the amino acid sequences of full-length PERVs and PERVs with one LTR

268 and at least one coding gene. All Gag, Pol and Env protein sequences (Dataset S1) were

269 aligned in MAFFT 7.222 and confirmed manually in MEGA7(42). The phylogenetic 
270 history of these gammaretroviruses was then determined using the maximum likelihood

271 (ML) method available in PhyML 3.1(40), incorporating 100 bootstrap replicates to assess

272 node robustness. The best-fit JTT $+\Gamma$ amino acid substitution model was selected for Gag,

273 Pol and JTT+ + I for Env using the ProtTest 3.4.2(43).

275 Additional Files

276 Additional files 1. Fig. S1. Maximum likelihood (ML) tree of the 5' and 3' LTRs of all

277 full-length PERVs. Fig. S2. Detailed descriptions of eJJRV genome. Fig. S3. The

278 alignment of LTRs of PERVs and eJJRV. Fig. S4. The complete phylogenetic tree of Gag.

279 Fig. S5. The complete phylogenetic tree of Pol. Fig. S6. The complete phylogenetic tree of

280 Env. Fig. S7. The phylogenetic trees of Gag, Pol and Env of Muroidea ERVs, eJJRVs,

281 ePCRVs, PERVs and representative retroviruses.

282 Additional files 2. Table S1. The information of pig, rodent, and rock hyrax genomes

283 used for data mining. Table S2. The information of full-length and near full-length

284 PERVs. Table S3. The recombination-related information of full-length PERVs shown in

285 Fig. S1. Table S4. The information of 142 mammals used for PERV-like sequences

286 mining. Table S5. The information of genes flanking the eJJRVs and ePCRVS. Table S6.

287 The information of full-length and near full-length Muroidea ERVs. Table S7. The

288 information of representative retroviruses used for phylogenetic analysis.

289 Additional files 3. Data set S1. The alignments used to build the phylogenetic trees of

290 Gag, Pol and Env represented in Fig. S4, S5, and S6, respectively. Data set S2. The 291 alignments used to build the phylogenetic trees of Gag, Pol and Env represented in Fig. 
292 S7.

293

\section{Declarations}

295 Ethics approval and consent to participate. Not applicable.

296 Acknowledgement. Not applicable.

297 Funding. J.C. is supported by National Natural Science Foundation of China under grant 298 no. 31671324 and CAS Pioneer Hundred Talents Program.

299 Availability of data and materials. The dataset supporting the conclusions of this article

300 is described in the main text (and additional files)

301 Author contributions. J.C. conceived and designed the research. Y.C. and M.C.

302 conducted the analyses. X.D. participated in the analysis of full-length PERVs. J.C.

303 supervised the whole project. All authors participated in the project discussion and 304 manuscript preparation.

305 Competing interests. The authors declare that they have no competing interests

307 References

308 1. Ekser B, Cooper DKC, Tector AJ. The need for xenotransplantation as a source of

309 organs and cells for clinical transplantation. International journal of surgery (London,

310 England). 2015;23(Pt B):199-204.

311 2. Ekser B, Ezzelarab M, Hara H, van der Windt DJ, Wijkstrom M, Bottino R, et al.

312 Clinical xenotransplantation: the next medical revolution? Lancet (London, England). 313 2012;379(9816):672-83. 
314 3. Niu D, Wei HJ, Lin L, George H, Wang T, Lee IH, et al. Inactivation of porcine 315 endogenous retrovirus in pigs using CRISPR-Cas9. Science (New York, NY). $3162017 ; 357(6357): 1303-7$.

317 4. Denner J. Paving the Path toward Porcine Organs for Transplantation. The New 318 England journal of medicine. 2017;377(19):1891-3.

319 5. Wegman-Points LJ, Teoh-Fitzgerald ML, Mao G, Zhu Y, Fath MA, Spitz DR, et al. 320 Retroviral-infection increases tumorigenic potential of MDA-MB-231 breast carcinoma 321 cells by expanding an aldehyde dehydrogenase (ALDH1) positive stem-cell like 322 population. Redox biology. 2014;2:847-54.

323 6. Denner J, Young PR. Koala retroviruses: characterization and impact on the life of 324 koalas. Retrovirology. 2013;10:108.

325 7. Aiewsakun P, Katzourakis A. Marine origin of retroviruses in the early Palaeozoic 326 Era. Nature communications. 2017;8:13954.

327 8. Denner J, Tonjes RR. Infection barriers to successful xenotransplantation focusing on 328 porcine endogenous retroviruses. Clinical microbiology reviews. 2012;25(2):318-43.

329 9. Tonjes RR, Niebert M. Relative age of proviral porcine endogenous retrovirus 330 sequences in Sus scrofa based on the molecular clock hypothesis. Journal of virology. $331 \quad 2003 ; 77(22): 12363-8$.

332 10. Morozov VA, Wynyard S, Matsumoto S, Abalovich A, Denner J, Elliott R. No PERV 333 transmission during a clinical trial of pig islet cell transplantation. Virus research. $334 \quad 2017 ; 227: 34-40$.

335 11. Crossan C, Mourad NI, Smith K, Gianello P, Scobie L. Assessment of porcine 
336 endogenous retrovirus transmission across an alginate barrier used for the encapsulation of

337 porcine islets. Xenotransplantation. 2018:e12409.

338 12. Wynyard S, Nathu D, Garkavenko O, Denner J, Elliott R. Microbiological safety of

339 the first clinical pig islet xenotransplantation trial in New Zealand. Xenotransplantation.

$340 \quad 2014 ; 21(4): 309-23$.

341 13. Moalic Y, Blanchard Y, Félix H, Jestin A. Porcine Endogenous Retrovirus Integration

342 Sites in the Human Genome: Features in Common with Those of Murine Leukemia Virus.

343 Journal of virology. 2006;80(22):10980-8.

344 14. Czauderna F, Fischer N, Boller K, Kurth R, Tonjes RR. Establishment and

345 characterization of molecular clones of porcine endogenous retroviruses replicating on

346 human cells. Journal of virology. 2000;74(9):4028-38.

347 15. Patience C, Takeuchi Y, Weiss RA. Infection of human cells by an endogenous

348 retrovirus of pigs. Nature medicine. 1997;3(3):282-6.

349 16. Li Z, Ping Y, Shengfu L, Hong B, Youping L, Yangzhi Z, et al. Phylogenetic

350 relationship of porcine endogenous retrovirus (PERV) in Chinese pigs with some type C

351 retroviruses. Virus research. 2004;105(2):167-73.

352 17. Cui J, Tachedjian G, Tachedjian M, Holmes EC, Zhang S, Wang LF. Identification of

353 diverse groups of endogenous gammaretroviruses in mega- and microbats. The Journal of

354 general virology. 2012;93(Pt 9):2037-45.

355 18. Niebert M, Tonjes RR. Evolutionary spread and recombination of porcine endogenous

356 retroviruses in the suiformes. Journal of virology. 2005;79(1):649-54.

357 19. Scheef G, Fischer N, Krach U, Tonjes RR. The number of a U3 repeat box acting as 
358 an enhancer in long terminal repeats of polytropic replication-competent porcine 359 endogenous retroviruses dynamically fluctuates during serial virus passages in human 360 cells. Journal of virology. 2001;75(15):6933-40.

361 20. Wilson CA, Laeeq S, Ritzhaupt A, Colon-Moran W, Yoshimura FK. Sequence 362 analysis of porcine endogenous retrovirus long terminal repeats and identification of 363 transcriptional regulatory regions. Journal of virology. 2003;77(1):142-9.

364 21. Huh JW, Cho BW, Kim DS, Ha HS, Noh YN, Yi JM, et al. Long terminal repeats of 365 porcine endogenous retroviruses in Sus scrofa. Archives of virology. 2007;152(12):22713666.

367 22. Niebert M, Kurth R, Tonjes RR. Retroviral safety: analyses of phylogeny, prevalence 368 and polymorphisms of porcine endogenous retroviruses. Annals of transplantation. $369 \quad 2003 ; 8(3): 56-64$.

370 23. Mayer J, Blomberg J, Seal RL. A revised nomenclature for transcribed human 371 endogenous retroviral loci. Mobile DNA. 2011;2(1):7.

372 24. Johnson WE, Coffin JM. Constructing primate phylogenies from ancient retrovirus 373 sequences. Proceedings of the National Academy of Sciences of the United States of 374 America. 1999;96(18):10254-60.

375 25. Hughes JF, Coffin JM. Evidence for genomic rearrangements mediated by human 376 endogenous retroviruses during primate evolution. Nature genetics. 2001;29(4):487-9.

377 26. Lole KS, Bollinger RC, Paranjape RS, Gadkari D, Kulkarni SS, Novak NG, et al. 378 Full-length human immunodeficiency virus type 1 genomes from subtype C-infected 379 seroconverters in India, with evidence of intersubtype recombination. Journal of virology. 
1999;73(1):152-60.

27. Zhuo X, Feschotte C. Cross-Species Transmission and Differential Fate of an

382 Endogenous Retrovirus in Three Mammal Lineages. PLoS pathogens.

$383 \quad 2015 ; 11(11):$ e1005279.

28. Martoglio B, Graf R, Dobberstein B. Signal peptide fragments of preprolactin and

385 HIV-1 p-gp160 interact with calmodulin. The EMBO journal. 1997;16(22):6636-45. sequences of simian immunodeficiency viruses from chimpanzees in Cameroon are strongly related to those of human immunodeficiency virus group $\mathrm{N}$ from the same geographic area. Journal of virology. 2000;74(1):529-34.

390 30. Argaw T, Wilson CA. Detailed mapping of determinants within the porcine endogenous retrovirus envelope surface unit identifies critical residues for human cell

392 infection within the proline-rich region. Journal of virology. 2012;86(17):9096-104.

393 31. Watanabe R, Miyazawa T, Matsuura Y. Cell-binding properties of the envelope 394 proteins of porcine endogenous retroviruses. Microbes and infection. 2005;7(4):658-65.

395 32. Denner J. Recombinant porcine endogenous retroviruses (PERV-A/C): a new risk for 396 xenotransplantation? Archives of virology. 2008;153(8):1421-6.

397 33. Ericsson TA, Takeuchi Y, Templin C, Quinn G, Farhadian SF, Wood JC, et al. 398 Identification of receptors for pig endogenous retrovirus. Proceedings of the National 399 Academy of Sciences of the United States of America. 2003;100(11):6759-64.

400 34. Hayward A, Cornwallis CK, Jern P. Pan-vertebrate comparative genomics unmasks 401 retrovirus macroevolution. Proceedings of the National Academy of Sciences of the 
402 United States of America. 2015;112(2):464-9.

403 35. Harrison I, Takeuchi Y, Bartosch B, Stoye JP. Determinants of high titer in 404 recombinant porcine endogenous retroviruses. Journal of virology. 2004;78(24):13871-9.

405 36. Kaulitz D, Mihica D, Adlhoch C, Semaan M, Denner J. Improved pig donor screening 406 including newly identified variants of porcine endogenous retrovirus-C (PERV-C). 407 Archives of virology. 2013;158(2):341-8.

408 37. Altschul SF, Gish W, Miller W, Myers EW, Lipman DJ. Basic local alignment search 409 tool. Journal of molecular biology. 1990;215(3):403-10.

410 38. Xu Z, Wang H. LTR_FINDER: an efficient tool for the prediction of full-length LTR 411 retrotransposons. Nucleic acids research. 2007;35(Web Server issue):W265-8.

412 39. Ellinghaus D, Kurtz S, Willhoeft U. LTRharvest, an efficient and flexible software for 413 de novo detection of LTR retrotransposons. BMC Bioinformatics. 2008.

414 40. Guindon S, Dufayard JF, Lefort V, Anisimova M, Hordijk W, Gascuel O. New 415 algorithms and methods to estimate maximum-likelihood phylogenies: assessing the 416 performance of PhyML 3.0. Systematic biology. 2010;59(3):307-21.

417 41. Katoh K, Standley DM. MAFFT multiple sequence alignment software version 7: 418 improvements in performance and usability. Molecular biology and evolution. $419 \quad 2013 ; 30(4): 772-80$.

420 42. Kumar S, Stecher G, Tamura K. MEGA7: Molecular Evolutionary Genetics Analysis 421 Version 7.0 for Bigger Datasets. Molecular biology and evolution. 2016;33(7):1870-4.

422 43. Abascal F, Zardoya R, Posada D. ProtTest: selection of best-fit models of protein 423 evolution. Bioinformatics (Oxford, England). 2005;21(9):2104-5. 
426 Fig. 1 PERV proviruses in porcine genomes. (A) Genomic structure of PERVs,

427 including gag, pol and env genes and LTRs. Proviruses of PERV-A and PERV-A like (B),

428 PERV-B and PERV-B like $(\mathbf{C})$ and PERV-C and PERV-C like (D) groups depicted based

429 on reference PERV-A (accession number: AF435967.1), PERV-B (accession number:

430 EU523109.1) and PERV-C (accession number: HQ536015.1). LTRs of PERVs were

431 classified by the presence (type B) or absence (type A) of the 18 bp and 21 bp repeat

432 structure. Type B LTRs were divided into three subtypes (LTR B1, LTR B2 and LTR B3).

433 LTR A, B1, B2 and LTR B3 are presented in white, light gray, dark gray and black,

434 respectively. Insertions and deletions (< $50 \mathrm{bp}$ ) are depicted with blue and red flags,

435 respectively. Larger insertions ( $>50 \mathrm{bp})$ are labeled with green arrow. Large deletions $(>50$

436 bp) are shown without lines. Stop codons are showed with a black flag. (Du, Duroc pig;

437 Wu, Wuzhishan pig; El, Ellegaard pig; Ti, Tibetan pig; Go, Goettingen pig; Me, Meishan

438 pig; Ro, Rongchang pig; Ha, Hampshire pig; Lan, Landrace pig; Pi, Pietrain pig; Ba,

439 Bamei pig; Be, Bekshire pig; Lar, LargeWhite pig; Ji, Jinhua pig).

442 in eJJRV and PERVs. (B) Sliding window analysis of percent sequence identity along 443 pairwise alignments of proviruses without LTRs. Phylogenetic trees of Gag (C), Pol (D)

444 and Env (E) inferred using the amino acid sequences of PERVs, eJJRVs, ePCRVs and 445 other representative gammaretroviruses (Table S7). Bootstrap values $<70 \%$ are not shown 446 in phylogenetic trees. Trees were rooted to Reticuloendotheliosis virus (REV). The 
447 complete phylogenetic trees of Gag (C), Pol (D) and Env (E) are presented in additional

448 files 1: Fig. S4-S6, respectively. All abbreviations can be found in Table S7.

449

450 Fig. 3. Amino acid sequence comparison of the RBD of PERVs and ePCRVs. Two

451 ePCRVs (ePCRV_1(accession number: KN678005.1) and ePCRV_2 (accession number:

452 KN678690.1)) were aligned to PERV-A, -B, -C, and the newly discovered PERV-IM.

454 Fig. 4. Scenario of genesis of PERVs. A schematic representation of PERV evolutionary

455 history is shown, summarizing our hypothesis regarding the origin and evolution of

456 PERVs. Arrow on the left indicates putative timeline of each type of ERVs. Different

457 background colors illustrate putative evolution periods of modern PERVs. JJRV, PCRV,

458 ancestral PERV represent the exogenous forms of the retroviruses from their hosts. Solid

459 blue arrows show origination and dotted blue arrows show speciation. Abbreviation: RBD,

460 receptor binding domain; LTR, long terminal repeat; Gag, group antigens gene. 
pol

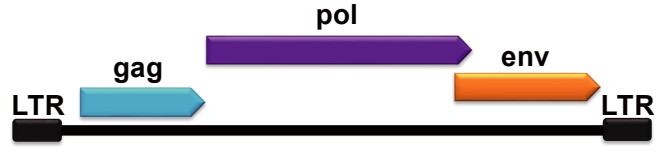

b

$\begin{array}{llllllllllll}\mathrm{Kb} & 0 & 1 & 2 & 3 & 4 & 5 & 6 & 7 & 8 & 9 & 10\end{array}$

Du_AEMK02000133.1

Du_AEMK02000536.1

Du_CM000812.5_C

Du_CM000812.5_b

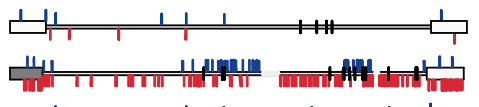

Du_CM000814.5_a $\square_{1,11}^{1} \stackrel{1}{=}$

Du_CM000816.5_b

Du_CM000817.5 开

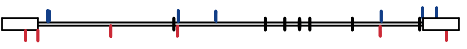

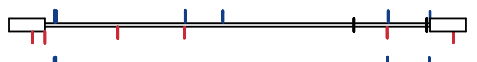
Du_CM000818.5_b $\square_{1}^{\frac{1}{1}}$

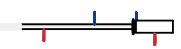

Du_CM000819.5_b

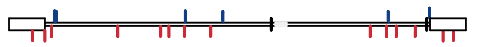

Du_CM000823.5_b 只

Du_CM000824.5_b

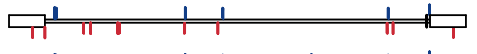

Du_CM000824.5_d |

Du_CM000826.5_a

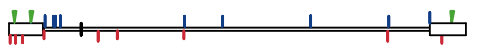
Du_CM000828.5_c

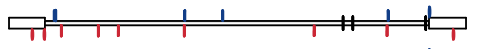
Du_CM000828.5_a Du_CM000830.5_c 厉 Du_LT634572.1_a

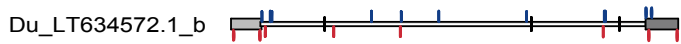

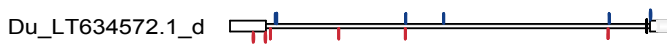

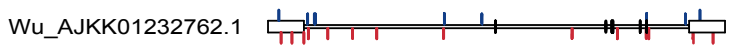
Ha_LUXS01031219.1 Ha_LUXS01060324.1 Pi_LUXU01038095.1

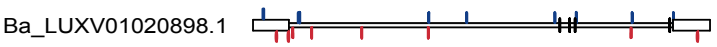
Be_LUXW01074277.1 Lar_LUXX01006697.1 Lar_LUXX01090830.1

Du AEMK02000137.1

Du AEMK02000197.1

Du_AEMK02000393.1_a

Du_AEMK02000476.1

Du_CM000812.5_a

Du_CM000814.5_c

Du_CM000815.5

Du_CM000818.5_a

Du_CM000819.5_a

Du_CM000820.5

Du_CM000822.5_b

Du_CM000823.5_a

Du_CM000825.5_b

Du_CM000826.5_b

Du_CM000827.5

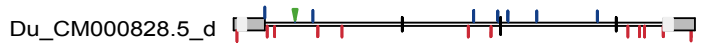

Du_CM000830.5_d

Ti_AORO02006834.1

Me_LUXQ01001340.1

Go_LIDP01000017.1

Ro_LUXR01004647.1

Ro_LUXR01022139.1

Ro_LUXR01063446.1

Ha_LUXS01013084.1

Pi_LUXU01008622.1

Ba_LUXV01061131.1

Lar_LUXX01045907.1

Lar_LUXX01080744.1

Ji_LUXY001101100.1

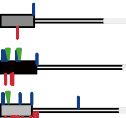

\section{(1)}

mil

d

$\begin{array}{llllllllllll}\mathrm{Kb} & 0 & 1 & 2 & 3 & 4 & 5 & 6 & 7 & 8 & 9 & 10\end{array}$

Du_CM000825.5_a

Wu_KQ003791.1 F

EI_AOCR01017097.1 بـ

EI_AOCR01079940.1 沜而

El_AOCR01098665.1 Tात

EI_AOCR01214680.1 $\mathrm{F}$ 项

ק

Ro_LUXR01088996.1

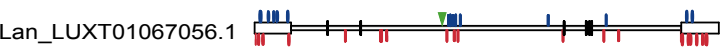

Pi_LUXU01026052.1 F

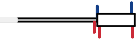
I Insertion
I Deletion
$\square \quad$ LTR A
$\square$
LTR B2
I Large Insertion I Stop codon
LTR B1 $\quad$ LTR B3 


\section{$a$}

eJJRV 5' LTR eJJRV 3' LTR PERV LTR B3 PERV LTR B2 PERV LTR B1 PERV LTR A

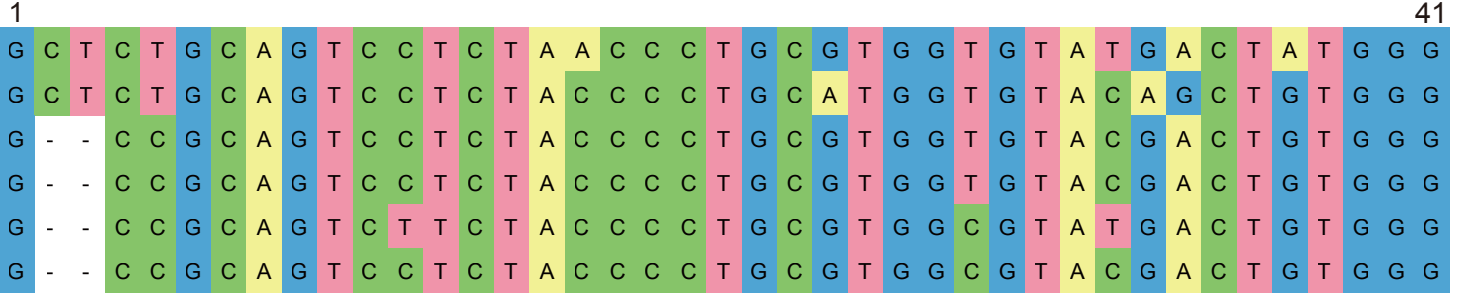

42

81

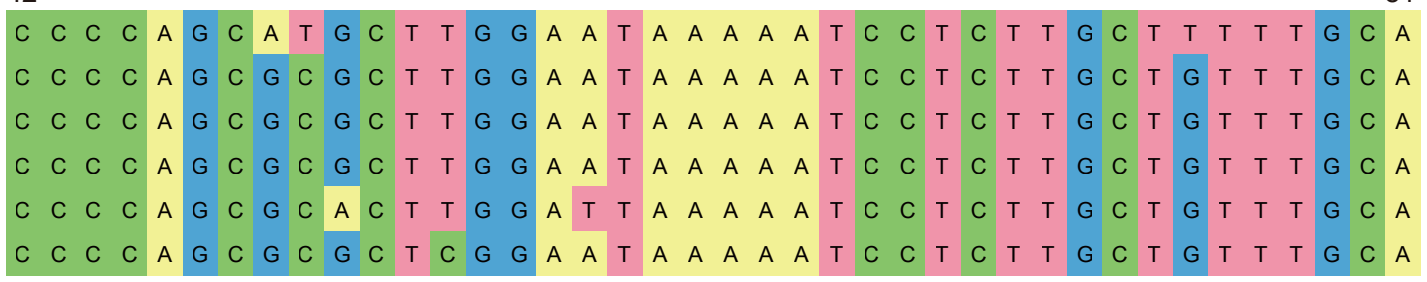

b

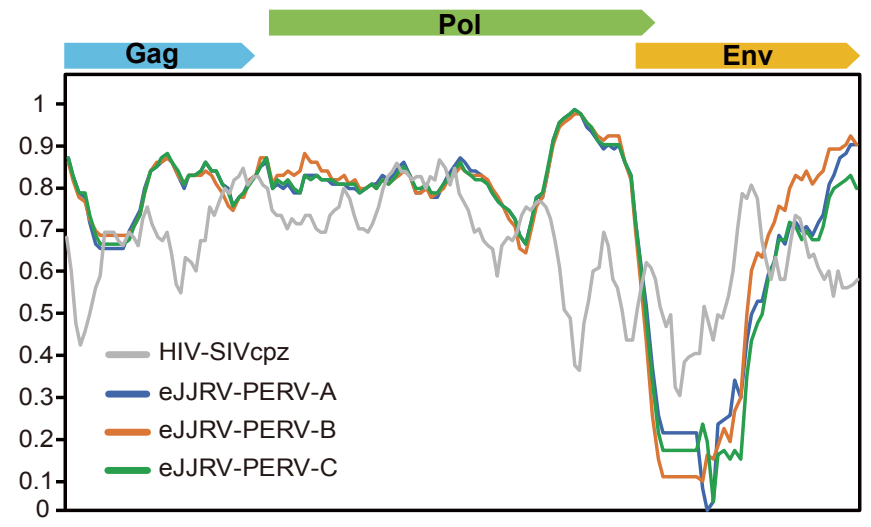

d
C
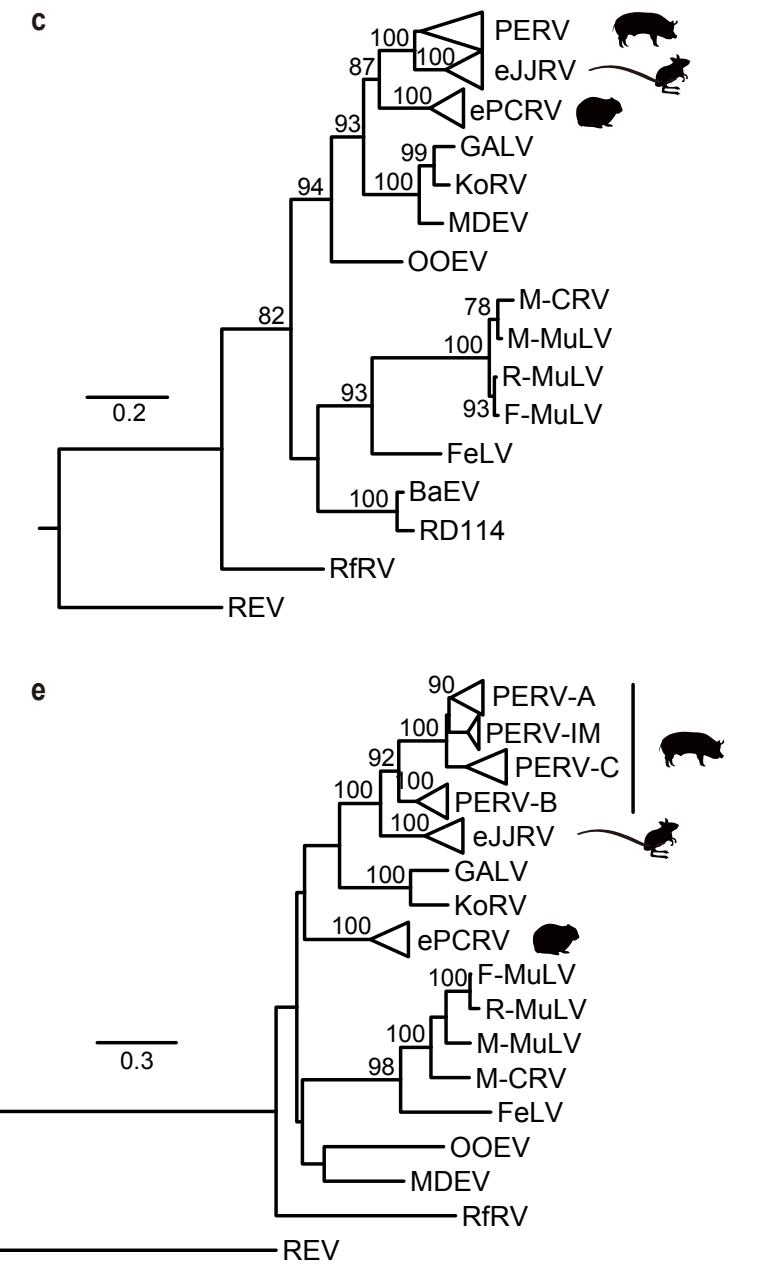
ePCRV_1 ePCRV_2 PERV-B PERV-A PERV-IM PERV-C

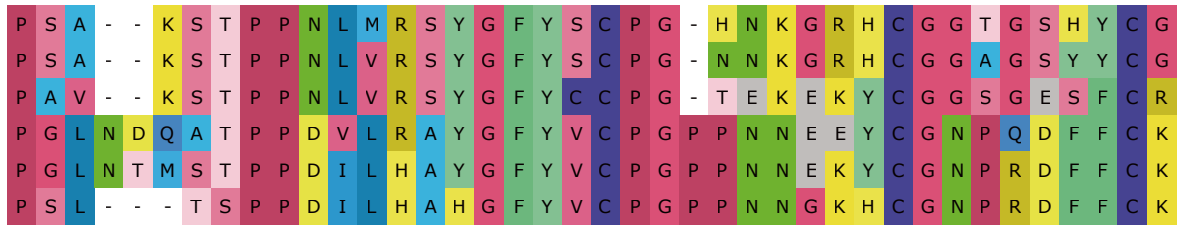

41

S W S C V T S N D G D W K W G I R K Q D L L K F S F V N P G P G K H V T M E SW S C V T S N D G D W KW G V G N R D L L K F S F V N P G P G K H V T M E $R$ W S C V T S N D G D W KW P I S L Q D R V K F S F V N S G P G K Y K V M K Q W S C I T S N D G N W K W P V S Q Q D R V S Y S F V N N P T S Y N N Q F N EW S C V T S N - G R Y KW P T S R Q D R A S F S F V N N Y T S S D Q L N Y Q W N C V T S N D G Y W K W P T S Q Q D R V S F S Y V N T Y T S S E Q F N Y - .

81

120

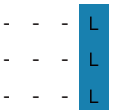

G R W K D W Q Q R V Q K D V R N K Q I S C H S L D L D Y L K I S F T E K G K Q E - - L LW - - - Q R E G K Y K Q I S C S P S D L D Y L K I S F T E K G E Q - - L L T - - - - - I R T G S P K C S P S D L D Y L K I S F T E K G K Q E

121

158

$P$ - S K W V D G L T W I V V W K Y S R G E - G S G L H V R L N I KR - - P $T-S K W I N G L T W G V V F W K Y G G G V-G S S L H I R L K I E S-P$

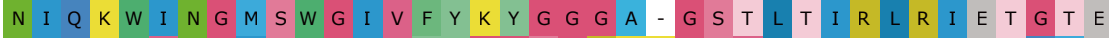

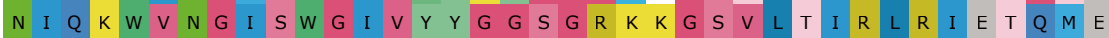
$N I-K W I N G M S W G I V F Y R G S G E$ I $-R$ S I L T I H L R I E T $Q$ M

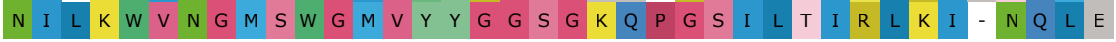




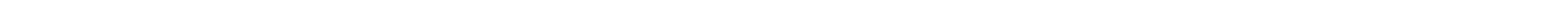

